\title{
Astrophysics without Black Holes, and without Extragalactic Gamma-Ray Bursts \\ Or: What have we learned from all our recent observations, including the G-W Receptions, FRBs, and the EHT-Map? Do they fit into a consistent Scenario?
}

\author{
Wolfgang Kundt* \\ Argelander-Institut für Astronomie der Universität Bonn, Auf dem Hügel 71, D-53121 Bonn \\ E-mail: wkundtesatro.uni-bonn.de
}

\begin{abstract}
In disagreement with a lot of recent literature, I shall argue that only at least two (extragalactic) gravitational-wave signals have so far been (strong enough to be) correctly interpreted, and evaluated as emitted by the merging of a binary neutron-star system. All ( $>$ seven) others were emitted too far from our Milky-Way Galaxy to be convincingly interpreted. No further comparably energetic source types are expected; in particular, Black Holes do not qualify.

My conviction that Black Holes are inconsistent - even with our human existence - is detailed in section 2. In particular, in sections 3 and 4, I repeat my understanding of the (million of) 'galactic rotational motions' as blown not by their central SMBH, but rather by their central Burning Disk (=: BD), a most powerful nuclear reactor.

In section 5, I discuss a large number of errors which can crop up in the discussion of our observations unless they have been correctly interpreted via all the inorganic machines which are active around us, in our Universe.

Finally, in section 6, I update my (almost universal) model of 'stellar jets' to include 'quasar jets', launched by their (massive) central BD.
\end{abstract}

Multifrequency Behaviour of High Energy Cosmic Sources - XIII - MULTIF2019

3-8 June 2019

Palermo, Italy

\footnotetext{
* Speaker.
} 


\section{A short history of the Detection of Gravitational Waves}

In the 1962 book 'Gravitation, an Introduction to Current Research' - edited by Louis Witten - Felix A. Pirani writes on page 199 that 'the weakness of the gravitational interaction makes it exceedingly unlikely that gravitational radiation will ever be the subject of direct observation'. In particular, Joe Weber's vibrating metal cylinder - of length some $2 \mathrm{~m}$ - has been many times claimed, but has never been sensitive enough to detect a non-terrestrial signal.

At the 'Ninth Texas Symposium on Relativistic Astrophysics' - just before Christmas 1978, in Munich - Joe Taylor presented 'evidence for the existence of gravitational radiation' from the measurement of repeating radio pulses emitted by the binary pulsar 1913+16, on pages 442-446. During the four years 1975-1979 of his observations, this binary pulsar apparently lost kinetic energy of orbital motion at a steady rate, 'consistent with the predictions of General Relativity Theory'.

During the more than ten years to follow, the Munich group around Jürgen Ehlers tried to make numerical predictions for its morphology, in particular for its exact final shape of 'inspiral, plunge, merger, and ringdown', of expected durations $\left\{>10^{2} \mathrm{sec}, 1 \mathrm{sec}, 10^{-1} \mathrm{sec}, 10^{-2} \mathrm{sec}\right\}$, respectively, during which the two neutron stars were expected to approach and touch each other, partially explode, and partially merge into a new, heavy neutron star plus ejected splinters. Their result will be discussed below, and is sketched in Fig.1; it is only marginally exact, but is expected to be a fair approximation of the gravitational wave emitted by such a contracting binary neutron-star system, except for its final $10^{-1.7} \mathrm{sec}$ before fusion, when its behaviour transcends all our attempts at an exact modelling.

An internet draft sent to me by Kip Thorne in February 2016, and referred to John A. Wheeler ${ }^{1}$, predicts a spiral-in time of 3.02 $10^{8} \mathrm{yr}$ for the Hulse-Taylor Binary Pulsar PSR 1913+16, a long time to wait. Depending on the number of similar binary pulsars in our Galaxy - probably not more than $10^{8}$ - we cannot expect more than one Galactic merger per year, rather less than one Galactic merger per $10^{3} \mathrm{yr}$, so that most present-day GW events should only come from such systems in more distant galaxies, like GW 170817 and GW 250419 did, and like most likely most of the other (>6) detected GW events did so far. Here I express my belief that merging binary neutron stars are the only extragalactic events to be recorded by the Advanced LIGO family, for at least many decades to come, because there are no other unstable, heavy, rotating multiple-stellar systems expected to exist in our present cosmic neighbourhood: Among all the stars we know, only neutron stars are compact enough to liberate enough energy $\mathrm{E}$ during fusion, for as giant transient metric distortions as have been observed, some $\delta \mathrm{E} \approx G m^{2} / r \approx 10^{53.6} \mathrm{erg}$ for 2 merging neutron stars, more than the energy of a hundred Supernovae. Consequently, Advanced LIGO may even have reached (more or less) the goal of gravitational-wave observations, for a long time to come. Or does Rosa Poggiani's talk [these proceedings] contain further realistic candidates, whose measurability I have underestimated? Will extraterrestrial measurement device reach sensitivities which allow the detection of distinctly fainter signals?

Returning to Thorne's internet draft, what has been achieved during those years of worldwide numerical studies are the derivation of two handy formulae, via the effective-one-body formalism

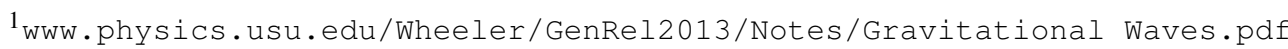


of Landau \& Lifschitz II combined with Thibault Damour's endeavours, which have yielded the approximate equation of motion:

$$
M_{c}:=\frac{\left(m_{1} m_{2}\right)^{3 / 5}}{\left(m_{1}+m_{2}\right)^{1 / 5}}=\frac{c^{3}}{G}\left[\frac{-5}{2^{13 / 3}}\left(\omega(t)^{-8 / 3}\right)\right]^{3 / 5},
$$

involving the (constant) 'chirp mass' $\mathrm{M}_{\mathrm{c}}$ which determines the two merging masses $\mathrm{m}_{\mathrm{i}}$; where $\omega=$ $\omega(t)$ is their (chirping) angular velocity around each other, which rises roughly as $(\Delta t)^{-1 / 2}$ with time t during (evaluable) approach. And which has also yielded the luminosity-distance formula

$$
d=\frac{5}{24} \frac{c}{h_{0}} \frac{\dot{\omega}}{\omega^{3}}
$$

for the emitting binary system, $\mathrm{h}_{0}=\mathrm{h}_{0}(\mathrm{t})$ being its (varying) gravitational-wave amplitude. Note that for $\mathrm{m}_{1}=\mathrm{m}_{2}=: \mathrm{m}$, the chirp mass $\mathrm{M}_{\mathrm{c}}=2^{-1 / 5} \mathrm{~m} \simeq 0.8 \mathrm{~m}$ roughly equals $\mathrm{m}$. Required for these determinations is a reliable measurement of its chirp $\dot{\omega}(t)$ during its ultimate minute of inspiral. On the other hand, no exact formula has been derived ever that would have quantitatively described the complete (variable) spacetime geometry around a collapsing stellar binary, and the implied structure of its emitted wave, during its final hundredth of a second.

With this insight, have we reached a complete qualitative understanding of gravitational-wave astrophysics? Not really. In particular, GW 170817 was not only seen at gravitational waves, but also at various electromagnetic waves - if only weakly so - because of its gigantic distance from us - of $40 \mathrm{Mpc}$ - and only for comparably short times. For instance, at $\gamma$-rays, GW 170817 was seen somewhat later than its merging event - viz. starting 1.7 seconds later - and only measurable for comparably short times, because in order to be visible by us, the fragments of the almost luminally exploding binary had to first fill a sufficiently large sphere around their center, for sufficient brightness of their emitted radiation, and should - on the other hand - not have diluted too far either, again for sufficient brightness.

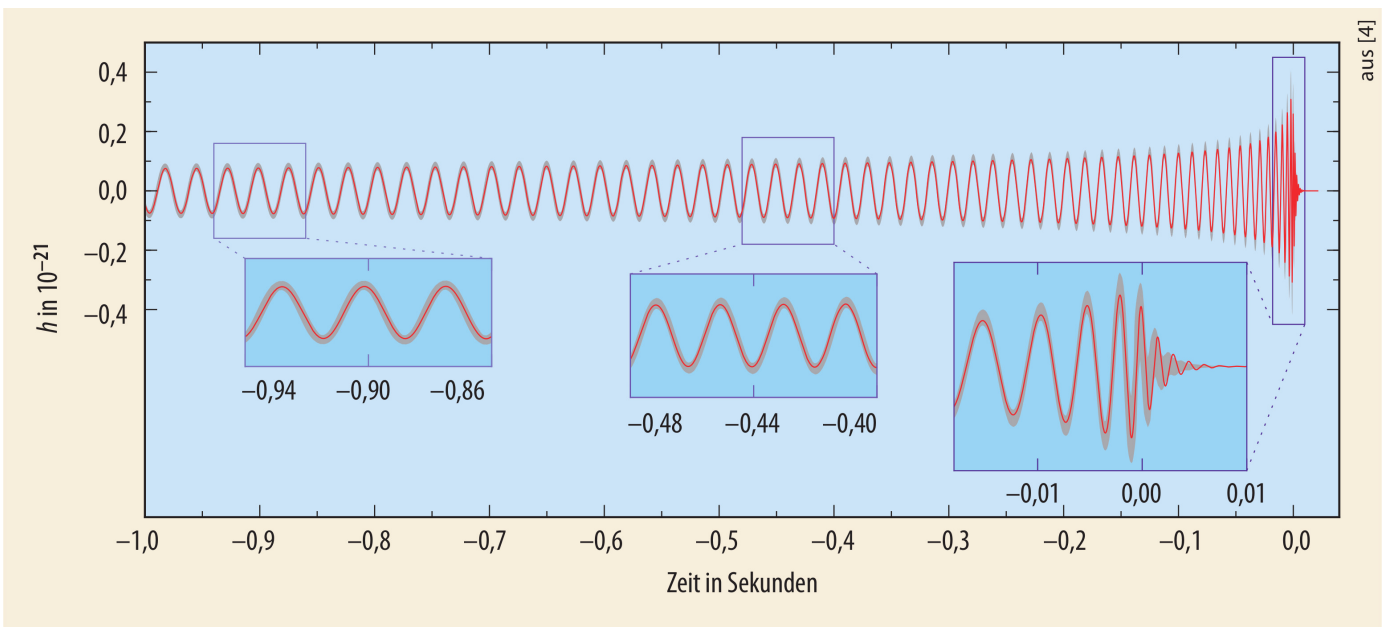

Figure 1: The last second of gravitational-wave radio signals observed by LIGO, and a few of its enlargements during the last few hundredths of a second, as calculated in Eq. 1.1. [from Schaefer, 2017] 


\section{Why should we dismiss singular structures, like Naked Singularities, or Black Holes?}

Among the alternative proposals of the observed GW events, Black Holes (BHs) have taken the first rank. But BHs were, so to speak, dead-born children: They were proposed - by Stephen Hawking and by his ph.d. father Roger Penrose - as the only regular collapse solutions of Einstein's GR equations - regular for their outside world - whose properties outside their horizon could therefore be rigorously calculated. They should not have been seriously considered anyway, however, because - as has been shown only a few years after their baptisation, in 1971 - they form a subset of measure zero within the set of all collapse solutions, i.e. are physically unexpected [Joshi, 2009, 2013]. Besides, singular solutions would not have made these alternative interpretations particularly attractive either, for our quantitative physical description. See also Hoyle et al [2000], as non-proponents.

Still, returning once more to $\mathrm{BHs}$ - inspite of their popularity, and in particular by having been financially well supported - BHs have failed to satisfy our physicists' desires, because throughout some five decades, all proposed candidates in the sky have been replaced by regular, even more plausible solutions of Einstein's GR theory. No need for BHs, or for NSs, and no evidence for them either, only ample financial support [Kundt, 1998, 2015, 2018]. Even worse: all of us have underestimated their quasi-exponential, chaotic growth - via accretion - in mass and, consequently, in attractive force. At least one of them would long since have swallowed our solar system, including ourselves - most likely (the BD, declared SMBH) Sgr A*, at our Galactic center - already within some $10^{8} \mathrm{yr}$ after its formation; isn't that a strong enough argument against its existence ?!? When Advanced LIGO (2016) advertised to have detected mergings of BHs of several solar-masses in the sky, several times in a row, these were false claims. Their falseness was already evident by claiming to have measured their spins as well, an inconsistent statement, too many parameters: two masses, two spins, plus their distance from us, determined from the set of measured data, less than the final $10^{-1.7} \mathrm{sec}$ of their chirp. Not even the $10^{2} \mathrm{sec}$ chirp of GW 170817 has allowed a determination of more than two (merging) masses plus their distance from us.

A much simpler explanation of the first six GW events from Advanced LIGO is that their signals have reached us from even more distant galaxies (than the signal GW 170817); they were, therefore, too weak for having been meaningfully evaluated, and should be forgotten.

And the claim by the team of the EVENT HORIZON TELESCOPE - on 10 April 2019 - to have mapped a SMBH at the center of the giant elliptical (and radio) galaxy M87, at the (single) radio wavelength of $1.3 \mathrm{~mm}$ - via its 'shadow' - is no more than an artist's belief: What (hot) lamp was powerful and near enough to this center to have cast this 'shadow', of temperature $\gtrsim 10^{9.5} \mathrm{~K}$, from a distance of $17 \mathrm{Mpc}$ ?! Maps of this same region in the X-ray sky - by CHANDRA - tell a different story: It's the (site of the) central engine of the twin-jet of M87, a Burning Disk [Kundt 1978, 1979, 1985, 1990, 1996, 2002, 2013, 2017a] - involving a nuclear reactor burning hydrogen into helium - that has blown this 'shadow', and is even strong enough to have blown its large twinjet - of size $\gtrsim 100 \mathrm{kpc}$ - like in all the other radio galaxies in our world-wide surroundings, whose masses have been carefully assembled in the SDSS plot, see: [Kormendy \& Bender, 2011], and: 4MOST Consortium Survey 6: AGN, in [The Messenger 175 (2019), p.43]. Alexander Unzicker agrees with my interpretation, also Yvan Leblanc, and Rainer Burghardt [2017], also Abhas Mitra, 
and Stanley Robertson, though not (yet) Harald Lesch, or Martin Gaskell, or the team that has successfully constructed and evaluated the EHT, or Bryan Gaensler (2011). There is no unanimosity (yet) worldwide on this crucial (non-) existence of SMBHs, which would meanwhile - if real have swallowed their surroundings in wide regions of our Universe, including our solar system, and ourselves.

Bye-bye, Black Holes!

\section{The spectrum of Sgr A*}

Sgr A*, the massive, unresolved point source at the center of our Milky Way galaxy, is much brighter, much harder in spectrum, and much more variable than is commonly stated, even though it looks comparable in appearance on most maps with its brightest surrounding stars. It is a Burning Disk, a =: BD. It is the extreme opposite of a SMBH. Of course, sources near the centers of galaxies suffer the largest foreground absorptions, i.e. look dimmer. And in addition, for fountain-like motions of their embedding medium, the escaping radiation must radially post-accelerate all the escaping winds, and ejecta, which it overtakes, and thereby get partially absorbed, and redshifted, hence strongly weakened: sources near deep potential minima look much dimmer than elsewhere. I have not seen a publication that had solved this problem self-consistently, but offer a new one in Fig. 2 with a collected spectrum that extends from $\mathrm{GHz}$ to $\mathrm{PeV} / \mathrm{h}=10^{30} \mathrm{~Hz}$, i.e. through some 21 orders of magnitude in frequency. It's the spectrum of a BD, an explosively nuclear-Burning Disk, as defined in [Kundt, 1978]. It is gigantic.

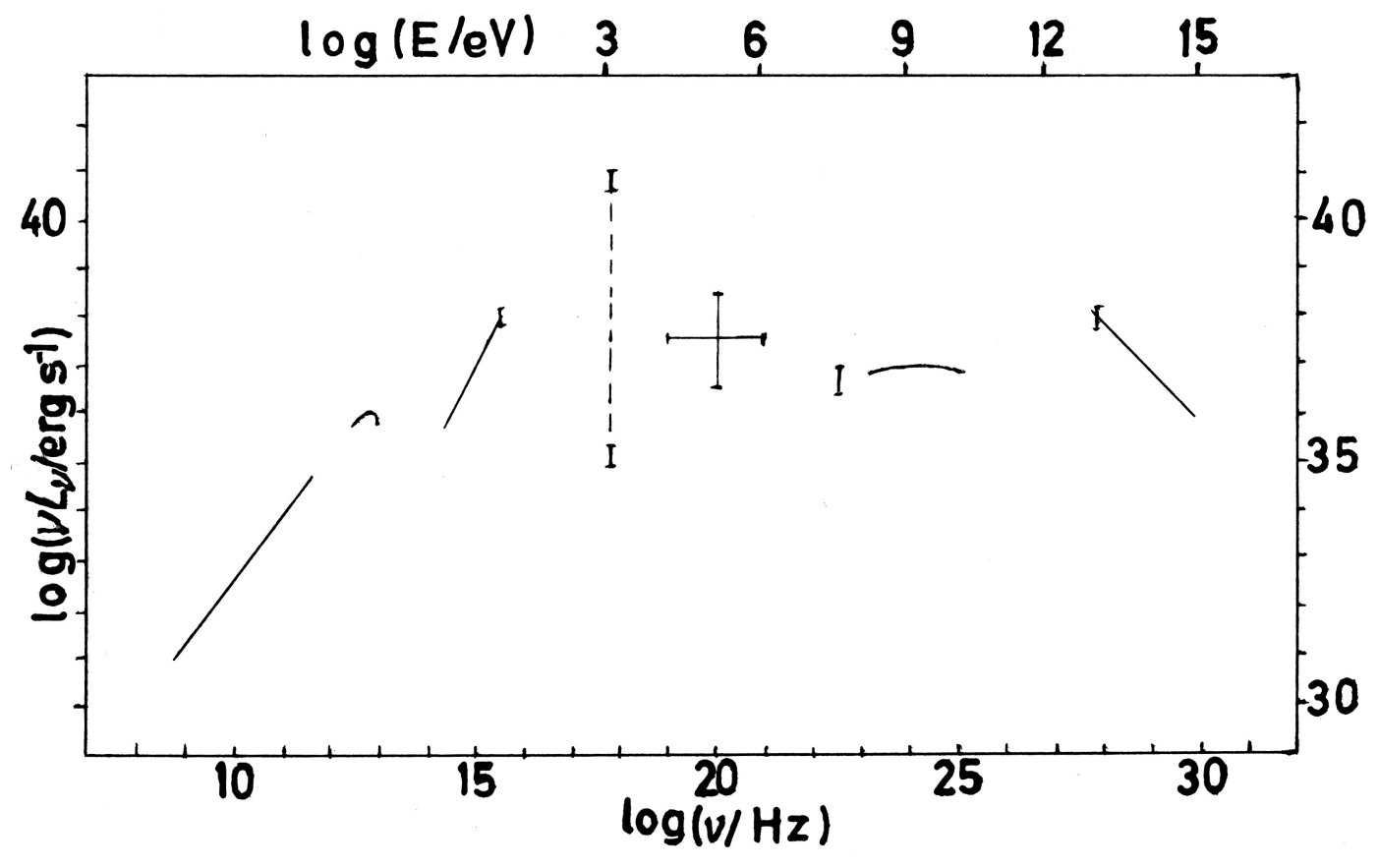

Figure 2: Best guess of an inferred spectrum of Sgr A*.

A SMBH could not emit this spectrum, as it swallows, rather than blows; its forces are inwardbound, of the wrong sign. 


\section{The rotational motions of galactic matter}

We now offer a (unique) solution for the controversial problem of what processes happen right infront of our cosmological doors, during the evolutions of all the galaxies around us. Their mass spectra have been published in the SDSS, updated in [Kormendy \& Bender, 2011], and more recently in: the Messenger No.175 (2019), some 100 times enhanced, if you rename their 'SMBHs' 'BDs', =: Burning Disks. As is well explained in [Ponti et al, 2019] for our Milky Way, and in a certain way even for all galaxies around ours already in [Kundt \& Krause, 1985], and again in [Kundt, 2017a], they show that matter inside the galactic disks spirals inward all the way to their centers, and is subsequently re-ejected - more or less explosively - through their halos via their BLRs, NLRs, EELs, ELRs, and/or jets towards their surroundings, via hot plasma streams; see also [Kundt 1978, 1979, 1985, 1990,1996, 2002, 2013, 2017a, 2020]. I.e., motion of matter in the disks of galaxies happens as (spiralling) infall, followed by stormy re-ejection. Note that if you replaced the central BD by a (swallowing) SMBH, something quite different would happen: ejection would be replaced by (singular) removal, contrary to observation.

The following five figures focus on Sgr A*, the BD of our Milky Way (in our interpretation), in steps of roughly factors of ten. They start (in Fig.3) by indicating the Fermi bubbles (purple contours) in its halo, and the (weak) Galactic jets: blue-shifted in the North, red-shifted in the South, and black their young, still line-less present-day replacement, jointly escaping from our Galaxy. Its disk is drawn in green.

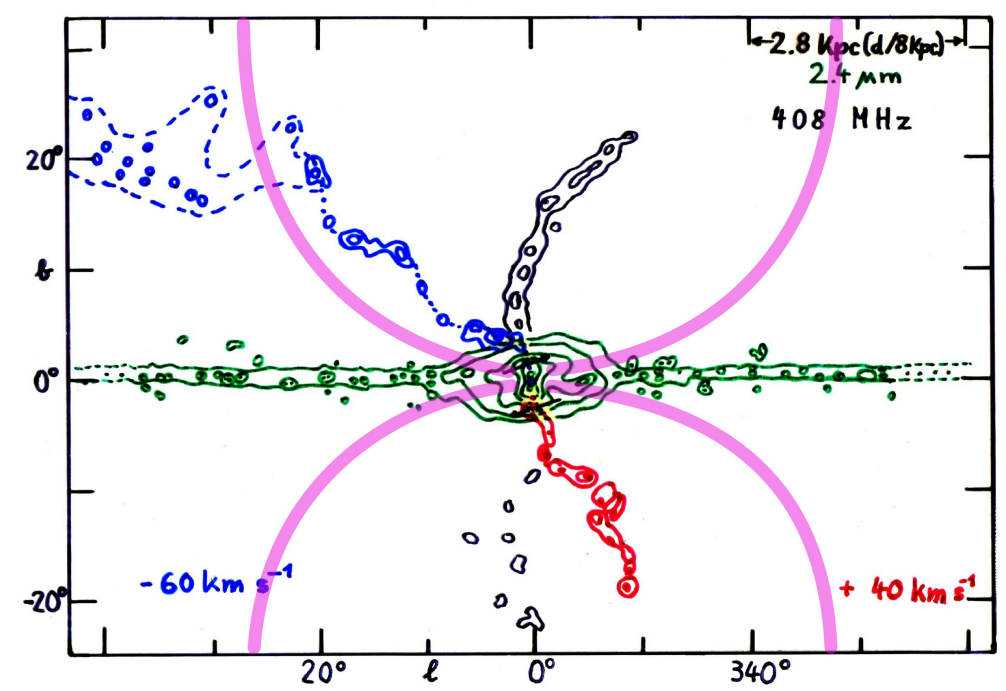

Figure 3: Remnants of the Galactic twin-jet and its observed radial velocities, and of the (pressurized) Fermi bubbles drawn in purple.

Next - in Fig.4 - traces of past 'chimneys' are drawn which are gleaned from radio maps, reminiscent of fluctuating ejection activity in the past.

Approaching the Galactic center by another factor of ten - in Fig. 5 - we recognise our Galactic twin jet during its past 300 years, including its central power house, Sgr A*. 


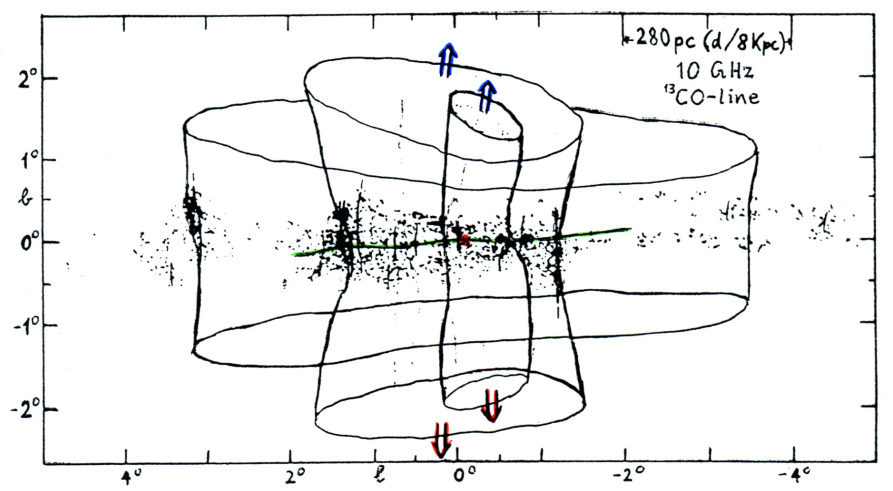

Figure 4: Polarised radio structure, enlarged w.r.t. Fig.3 by a factor of ten, gleaned from early radio maps.

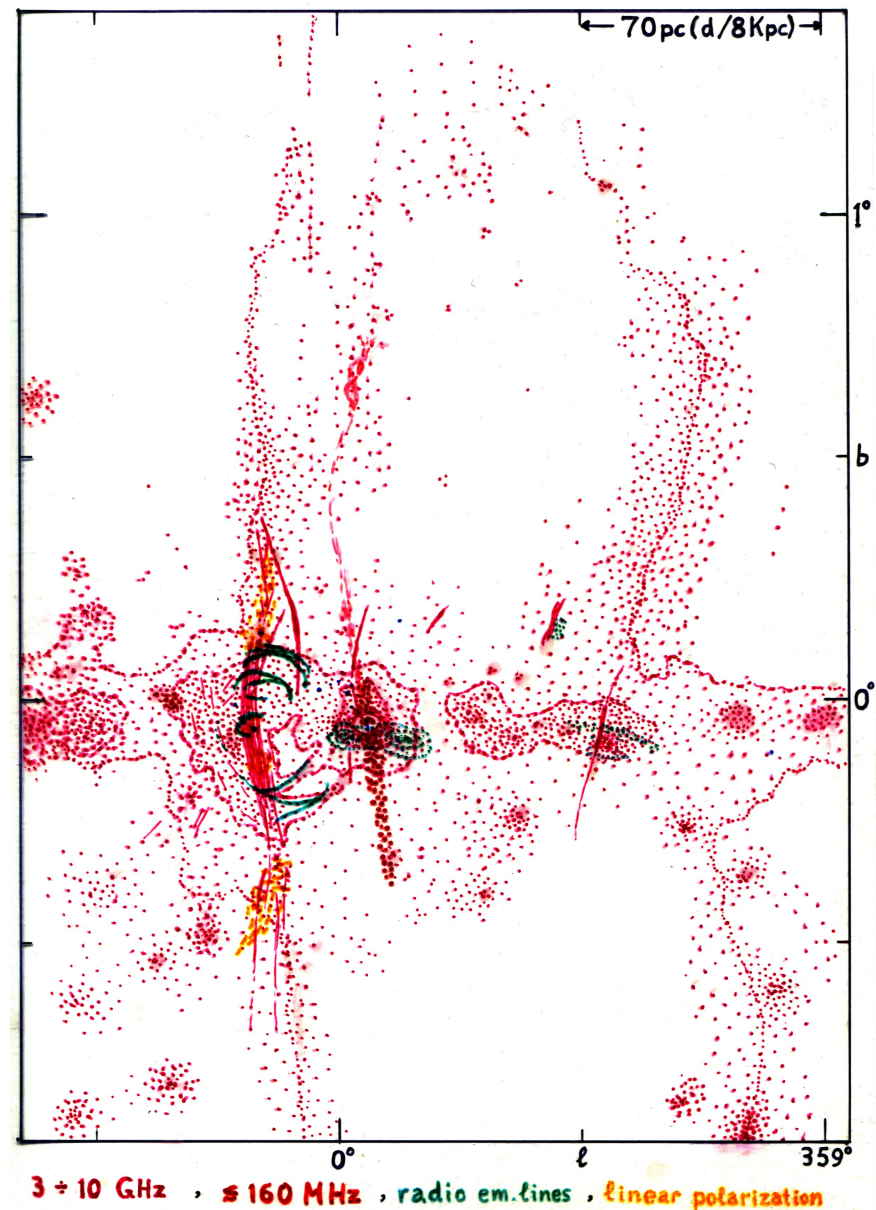

Figure 5: The innermost cylinder of Fig.4 looks like blown by the present-day jet from Sgr A*, located at longitude $-0.1 \mathrm{deg}$. Apparently, it is girdled by magnetic fields, revealed by its linear polarisation (drawn green and yellow). 


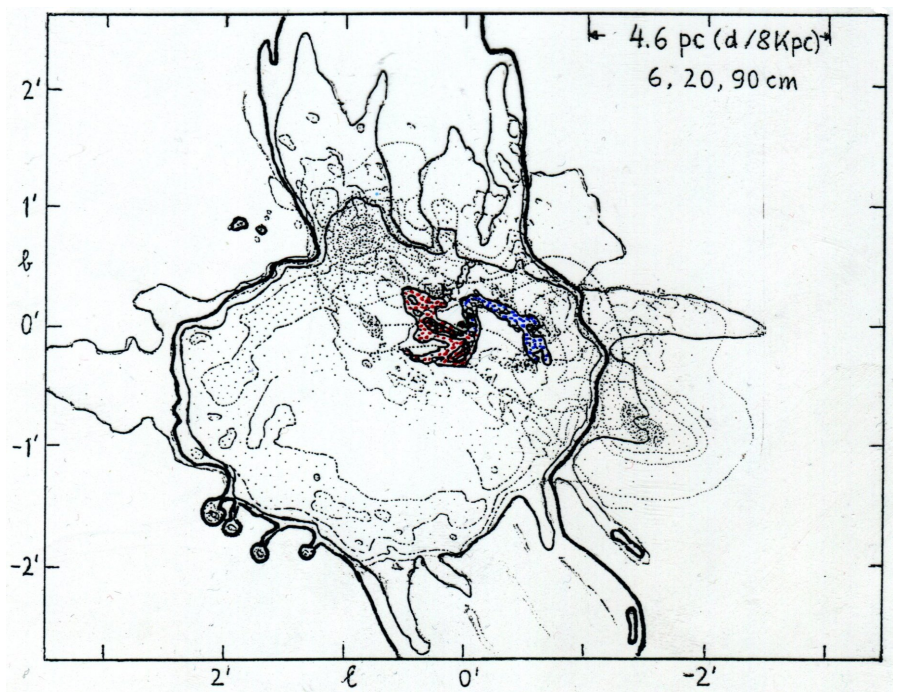

Figure 6: On the length scale of a few parsec, our Galactic center shows a high-pressure radio bubble Sgr A East - with outlets upward and downward in latitude, apparently to feed the Fermi bubbles with pressurized plasma which is probably injected by our Galaxy's BD, Sgr A*, the latter seen at the center of the mini-spiral Sgr A West.

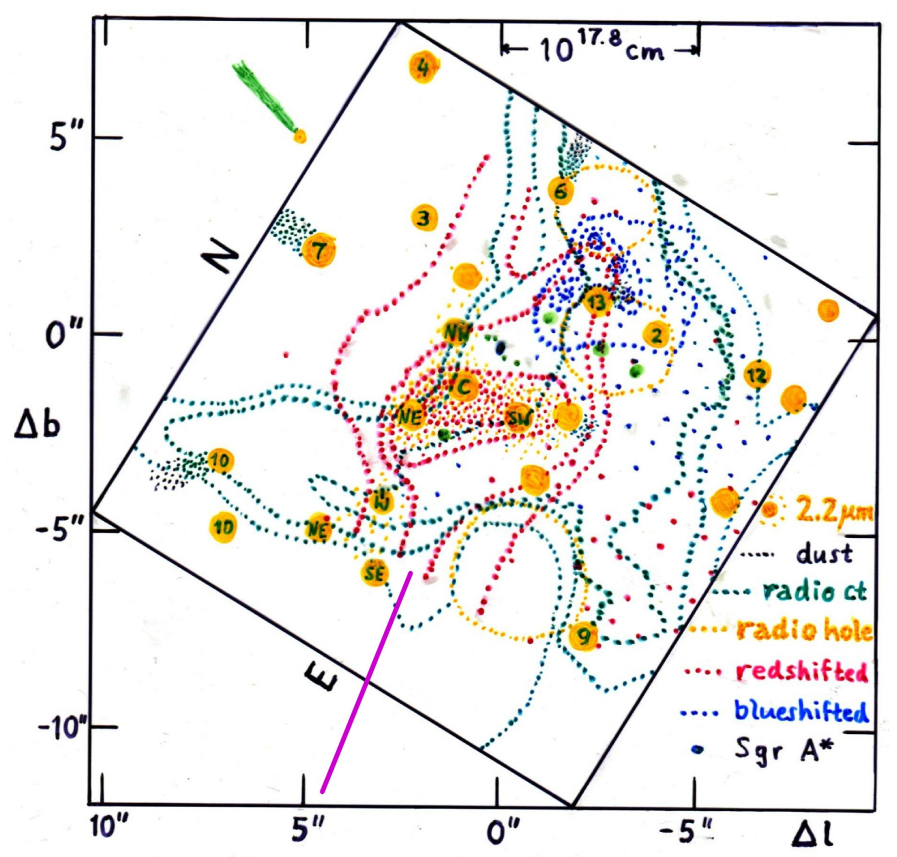

Figure 7: This innermost view of our Galactic center - centered by the unresolved point source Sgr A* carries signatures of a 2-sided outflow blown by it: its Broad-Line-Region. Note that the atmospheres of (more than) 8 stars are blown radially outward by it. The forming twin-jet of our Galaxy is indicated by purple pressure contours. 
Figure 6, for the first time, indicates a central storage bubble for blowing the northern and southern Fermi bubbles - Sgr A East - whose interior contains the central Galactic twin jet - first published by Frederick Baganoff et al at X-rays, in 2003 - not resolved in this figure.

In Figure 7, we recognise blueshifted and redshifted plasma currents on length scales of one lightyear, and even more than eight bright, close-to-center stars whose atmospheres are obviously blown off radially, away from Sgr A*. The twin jet is again unresolved. For more detailed interpretations, please consult reference [Kundt, 2017a].

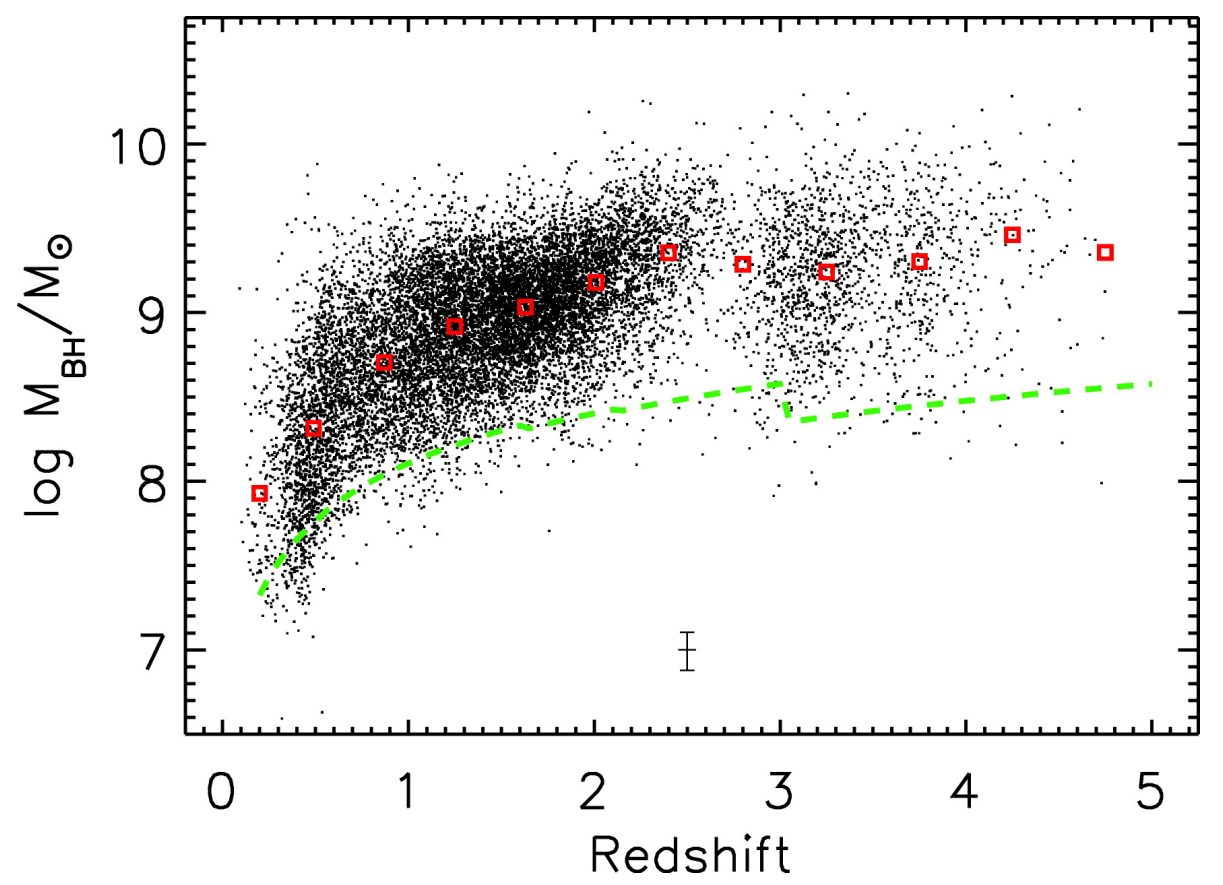

Figure 8: Measured masses, and unresolved core masses of some 15000 galaxies in the sky - by the SDSS - as explained in the text. Note that its incompleteness below redshifts $\mathrm{z} \lesssim 0.5$ has meanwhile been filled up by the quoted ESO plot, in the 2019 Messenger 175.

Figure 8 now shows the remarkable fact that more matter has been ejected statistically from the galactic centers than has spiralled in; that galaxies on average lose mass with time, consistent with what was found already in [Kundt \& Krause, 1985], via their Ly-alpha-forest spectra; the nuclear explosions inside the BDs are efficient, (as long as they contain enough (primordial) hydrogen). Again, SMBHs could not do that. And as has already been mentioned above - in section 2 - this important statistical result has been recently strengthened 100-fold in the Messenger 175 (2019), on page 43: The in-spiralling matter in galaxies is statistically more than re-ejected; (a bit) more matter is blown out than has fallen in. This important observational result agrees quantitatively with what had already been found in section 2 of ref.[17]: The 'Lyman alpha forests' around large galaxies reveal radially escaping matter - totalling $10^{10} \mathrm{M}_{\odot}$ in mass - at locally supersonic speeds. There is no gap in our understanding. 


\section{Further alternative interpretations}

In this talk, we have already encountered several controversial interpretations - in trying to understand GWs, and the EHT map - and the book by Ole Marggraf and myself [2014] contains more than further 135 of such 'alternatives', from the fields of physics, geophysics, astrophysics, and biophysics; my more recent papers [Kundt, 2015-2018] highlight some of the astrophysical ones at greater detail. It may therefore be helpful to find (below) the most frequent ones sorted out, and coarsely discussed.

Let us start with distance determinations. Large cosmic distances have often been inferred from (angular) coincidences with so-called 'host galaxies', most of which have later turned out to be fake. This problem began with the $\gamma$-ray bursts ( =: GRBs), and continued with the (more recent) fast radio bursts ( $=$ : FRBs), and introduced uncertainties (in distance) by factors of up to $10^{20}$, implying uncertainties in emission energy by factors of up to $\left(10^{20}\right)^{2}=10^{40}$, clearly inconsistent with (moderate) cosmic homogeneity, even with mere feasibility. They are hard-dies.

Another huge uncertainty of interpretations has entered astrophysics via the existence - or not - of BHs, or Naked Singularities, which have already taken a lot of space in this communication, but which are even harder-dies (than distance-uncertainties), hence will certainly survive for many more decades to come; cf. [Kundt, 2015].

A third, uncertain class of observations are the $G W s$, which form the preferred objects of this contribution. In my view, GW 170817 has been (until recently) one of the only two reliable events of this class, and is now (moderately) well understood.

Highly controversial is still the EHT single-radio-line, ultrahigh-resolution observation of the very center of the strong radio galaxy M87:

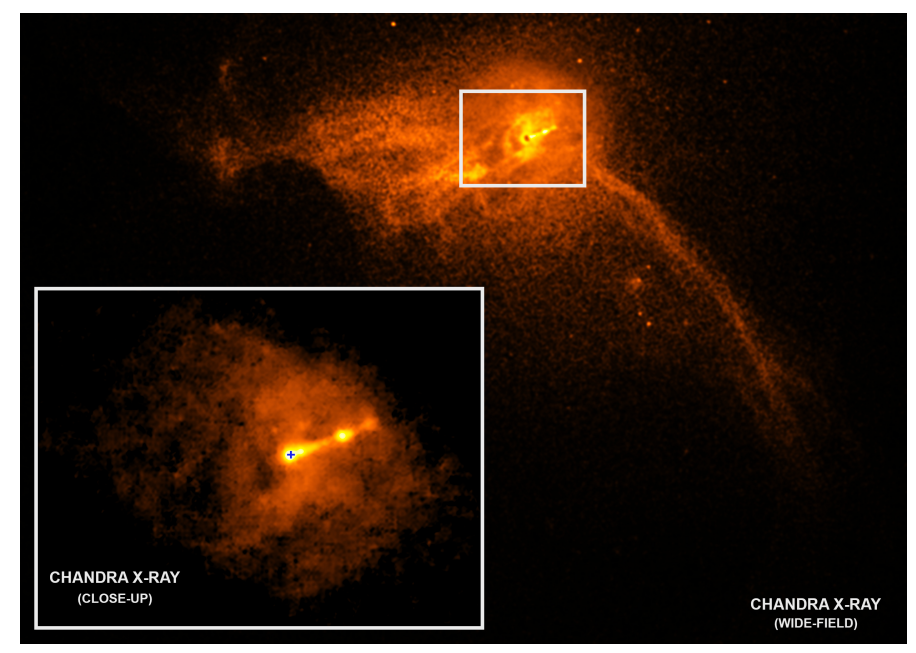

Figure 9: X-ray maps of the core region of the giant elliptical galaxy M87, at distance $17 \mathrm{Mpc}$. Note that the very center is extremely bright, probably due to its nuclear reactor, the $\mathrm{BD}$, which re-ejects its mass inflow; and that their unresolved central core region at $1.5 \mathrm{GHz}$ is of a (tiny) size of $0.1 \mathrm{lyr}$. - At Chandra's X-rays, instead, the unresolved core region is only as small as $300 \mathrm{lyr}$, some $10^{3.5}$ times larger, concluded from a width of 25.000 lyr of the lower side of the containing rectangula. 


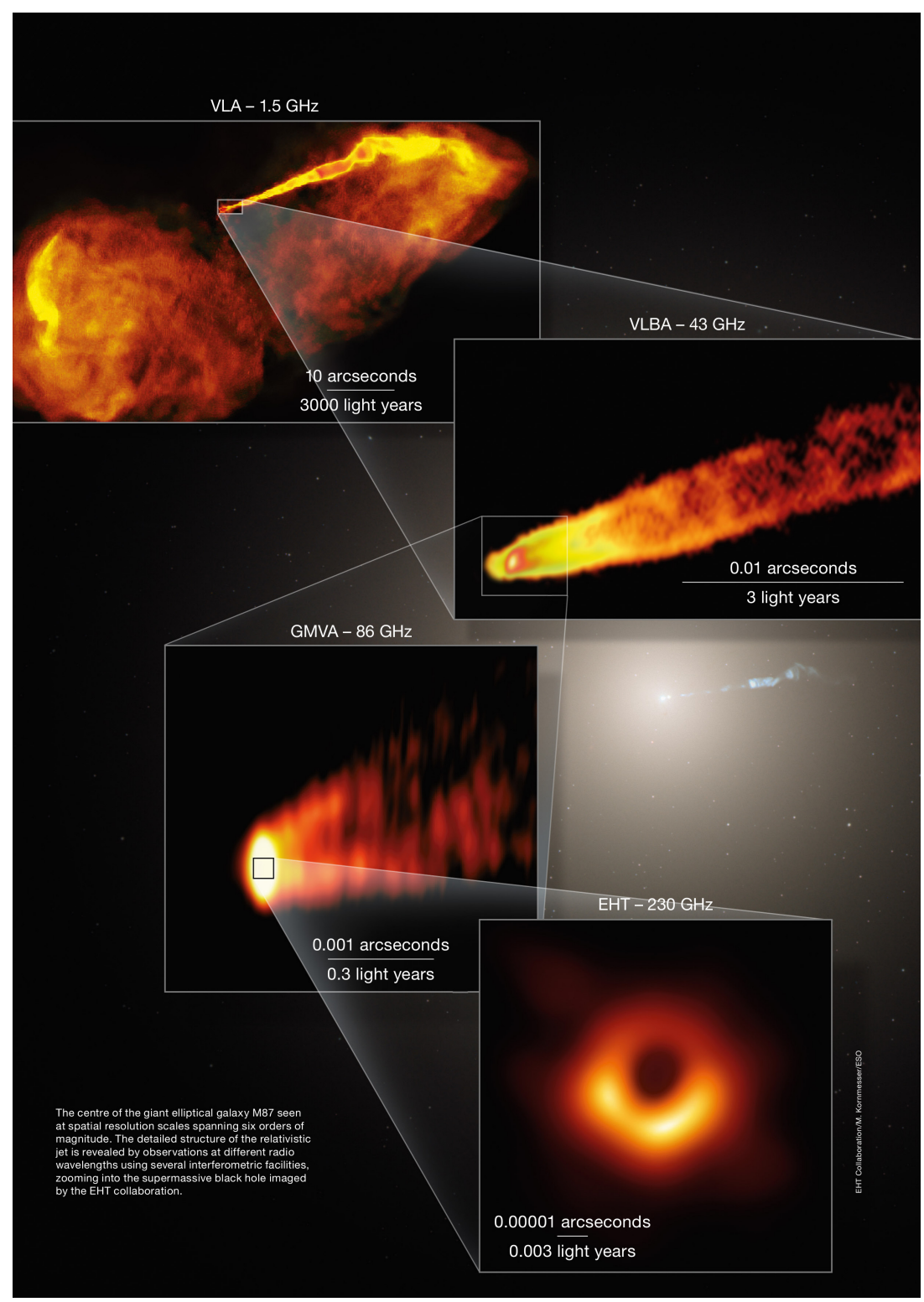

Figure 10: Radio maps of the core region of the giant elliptical galaxy M87, at distance $17 \mathrm{Mpc}$. Note that the very center is extremely bright and small, probably due to its nuclear reactor, the $\mathrm{BD}$, which re-ejects its mass inflow. The EHT map should have had this complete core-emission in its 8 beams, for sensitivity reasons alone.

Do we see its ejection- and jet-formation center, the more massive analog of Sgr A*, of effective temperature $\gtrsim 10^{9.5} \mathrm{~K}$, where its ejecta and pair plasma are formed and channelled, see Figs.9, 10 ? What lamp casts what radio shadow of what object? Isn't it simply the nuclear-burning matter supplied by the inward-spiralling disk - burning hydrogen to helium - plus its pair plasma formed by local coronal magnetic reconnections, a larger analog of Sgr A* in our Milky-Way galaxy ? At its huge distance (of $17 \mathrm{Mpc}$ ), only an extremely faint effective photon bundle could have reached the 8 telescopes of the (terrestrial) EHTelescope, of opening angle (Earth' diameter / distance) $=$ 
$10^{-17}$. Multiple photon squeezing and scattering - both internal and external - will have softened its spectrum during propagation, from $\mathrm{PeV}$ hardness down to $\mathrm{MeV}$ energies (via $\mathrm{e}^{ \pm}$-pair formation) and softer. What angular size has its effective source, at $1.3 \mathrm{~mm}$ ? For power reasons alone, the waning of the propagating signal - through a distance of $17 \mathrm{Mpc}$, by a factor of $10^{-34}$ - excludes all other known astrophysical sources as candidates, in particular ghost sources, like SMBHs.

The $F R B s$ are (likewise) still frontline objects. One of them - a peryton - was a little later found to be emitted by a stove in a nearby kitchen. All the others - in my view - come from the top of our mesosphere, created by (incoming) highest-energy cosmic-rays, colliding with the (sparse) local molecules and stripping off their electrons in the form of straight, charged sheets parallel to their incoming direction, which can mimic super-Galactic signals by creating (short-lived) large dispersion measures, DM $:=\int \mathrm{n} d \mathrm{~s}, \gtrsim 10^{2} \mathrm{~cm}^{-2}$, see Fig.11. Soon after their collision, (most of) their electrons are stopped and re-ejected (by the strong, local electric voltage, upward from our mesosphere), in the shape of the (multiply observed) terrestrial GRBs.

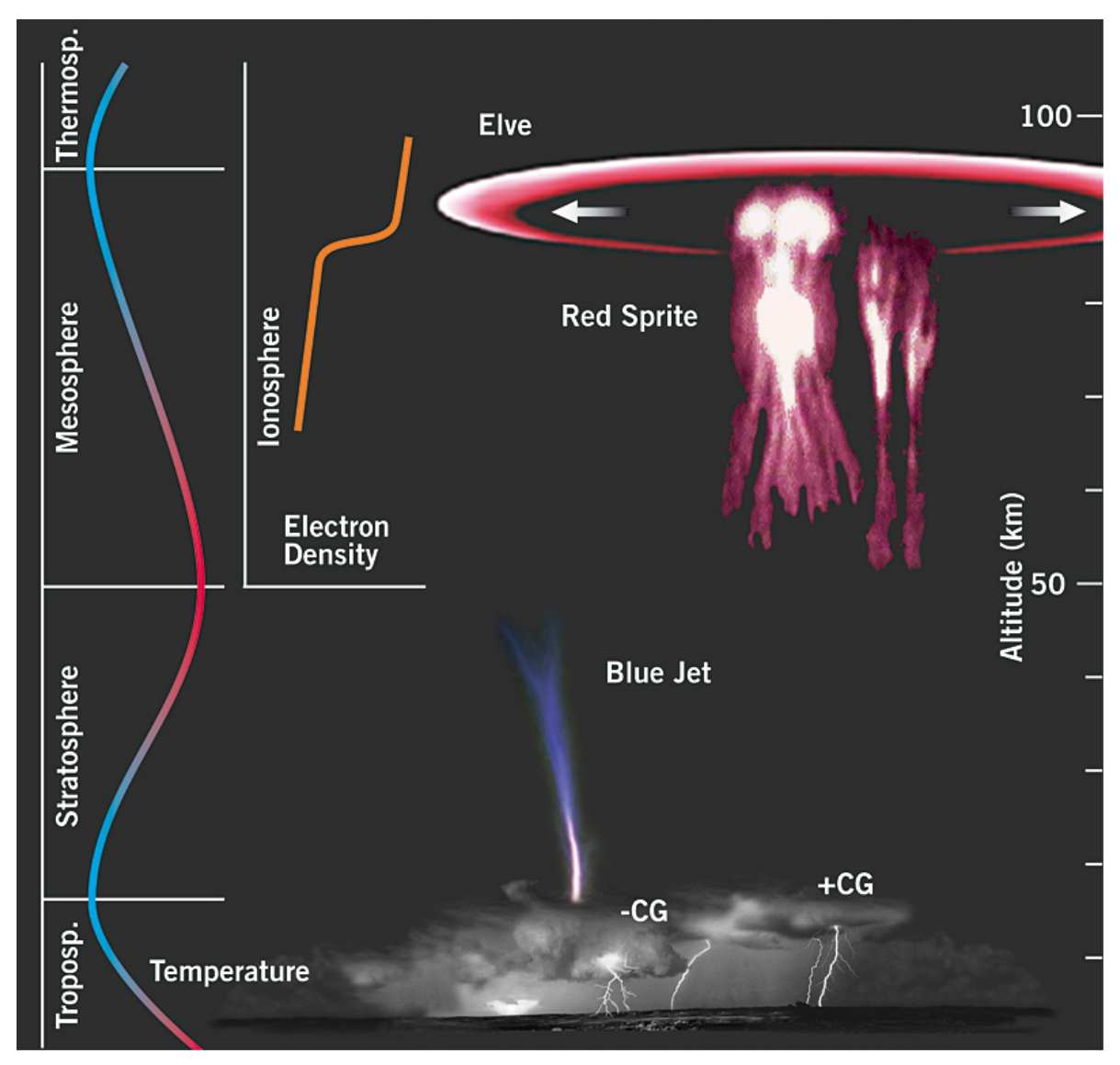

Figure 11: Sketch of the terrestrial atmosphere, at night.

A last word in this section may be spoken in concern with comparatively rare processes in the Universe, which function almost like manmade machines, or even like certain organs in biology, whose precise mechanisms we should understand before we can hope to model their operation, and reliability; let us call them 'inorganic machines'. Trying to model them by parameter-fitting, 
without a deep insight into their functioning, can give rise to very misleading results. Here I think in particular of the astrophysical jets of all (four) kinds [Kundt, 2017a], and next section, galactic rotations [Kundt, 2017b], supernovae, GRBs, (blowing) PSR winds [Kundt, 1998], PSR 'recycling', FRBs, forming the stars, planets, and moons (inside stellar disks), boosting the cosmic rays (=: $C R s$ ), creating thunderstorms, and many related phenomena. For a thorough understanding of them, we need quite specific education. In certain cases, a glance at [Kundt (1995, 2005), Kundt \& Marggraf, 2014] may help understand what I mean. But even here, I take the chance of this publication to update my own, multiply published jet model: it is too special for the (innermost parts of the) strong jets from quasars (by assuming them to be imbedded in vacuum), needs a slight generalization, which I have reserved for the next (and last) paragraph. Here it is:

\section{Quasar Jets}

For all the many jets which I have modelled in my life - with and without 'coats' - I assumed ambient vacuum, or at least approximate vacuum - an assumption which does not hold for the key source SS433, nor even approximately for the (strong) quasar jets, in the centers of active galaxies, where background pressures dominate in their galaxy. Their jets reach ambient (approximate) vacuum only at a certain distance downstream from their source, their galactic center, (of order $\gtrsim \mathrm{pc}$ ). They are born in extremely high-pressure, heavier regions. For them, the jet opening angle during propagation is initially quite large - larger than the typical $1 \%$ - as has recently been found by Yuri Kovalev et al by direct mapping, but shrinks continually with increasing length, until sufficiently low-pressure regions have been reached. Nevertheless, they are likewise (observed to be) supersonic - of Eilek type A - throughout some first $10^{5}$ years in the case of M87, like in the lower-density embedding media, just pay their friction credit to their high-pressure surroundings. Beyond, they are of Eilek type B.

In order to see this more clearly, note that the BD at the centers of active galaxies is not only an abundant producer of $\mathrm{e}^{ \pm}$pair plasma - via magnetic reconnections in its (top and bottom) magnetic halos - but at the same time a powerful nuclear reactor which re-ejects the incoming disk matter back towards its galaxy's periphery - initially through its BLRegion - an essentially hadronic plasma, some $10^{3.3}$ times heavier than electronic pair plasma. Whilst the (light) pair plasma is squeezed (up or down) essentially along the AGN's spin axis - via buoyancy - the much heavier ashes of nuclear burning fill a wide cone of ejection, surrounding its narrow, central twin jet. Initially, these surrounding ejection cones form high-pressure ambient mantles around the twin-jet, confining its trans-relativistic outward motion, and weakly braking it, via friction. Subsequently, however, the pressure of this ambient 'mantle' shrinks via expansion, and gradually drops to zero, leaving the enclosed twin-jet unconfined - like in all the other jet sources - and our earlier jet model applies without restriction.

Let me summarise the last two sections with a table called STRUCTURES, that lists these inorganic machines once more, together with some of their crucial properties which must not be ignored for successful models of their functioning. Here it is: 
Table 1: Table of STRUCTURES

\begin{tabular}{|c|c|}
\hline astrophysical jets are formed by & $\begin{array}{l}\text { \{young stars, white dwarfs, neutron stars, galactic } \\
\text { centers\}, surrounded by accretion disks }\end{array}$ \\
\hline rotating magnets with friction can create & trans-relativistic $\mathrm{e}^{ \pm}$-plasma \\
\hline galactic disks blow & galactic fountains \\
\hline Burning Disks re-eject infalling plasma & via explosive nuclear (H-) burning; e.g. Sgr A* \\
\hline Stellar disks form $\{$ stars, planets, moons $\}$ & via heavy clumping, guided by growth of entropy \\
\hline SN explosions & $\begin{array}{l}\text { require a magnetic piston, for reaching escape } \\
\text { speed from their star }\end{array}$ \\
\hline CR production & $\begin{array}{l}\text { via rare neutron-star accretion from its surround- } \\
\text { ing disk, and centrifugal re-ejection }\end{array}$ \\
\hline PSR-wind formation & via $\mathrm{HF} \beta \times B$-oscillations \\
\hline spinup of neutron stars to $\mathrm{P} \gtrsim 1.4 \mathrm{~ms}$ & via accretion from a surrounding disk \\
\hline GRBs & $\begin{array}{l}\text { from 'throttled' } \mathrm{n}^{* *}, \text { at } \lesssim \text { a few } 10^{2} \text { pc distance } \\
\text { from us, see light-echo ellipsoid }\end{array}$ \\
\hline FRBs & HE CR-impacts on upmost ionospheric molecules \\
\hline thunderstorms & HE impacts expel energetic electrons from cloud \\
\hline shadow of $\mathrm{BH}$ & see section 5 (on alternative interpretations) \\
\hline
\end{tabular}

\section{Acknowledgements}

My warm thanks go to Ole Marggraf, for help with the proceedings, and for regular, weekly conversations

\section{References}

[1] Abbott, B.P., et al. (LIGO Scientific Collaboration and Virgo Collaboration), Binary Black Hole Mergers in the First Advanced LIGO Observing Run, Phys. Rev. X 6, 041015, 2016.

[2] Burghardt, R.: Karl Schwarzschild und was mit seinem Werk geschah, Leibniz Online 26, 2017

[3] Hoyle, F., Burbidge, G, Narlikar, J., 2000: A Different Approach to Cosmology, Cambridge University Press, 357 pp.

[4] Joshi, Pankaj S.: Naked Singularities, the BH has a troublesome sibling, Scientific American, Febr. 2009 as well as: Vol. 22, No.2, May 21 (2013).

[5] Kormendy, John, Bender, Rolf: Nature 469, 374-379, 2011.

[6] Kovalev, Y.Y.,Pushkkarev, A.B.,Nokhrina, E.E.,Plavin, A.V.,Beskin, V.S., Chernoglazov, A.,Lister, M.L., Savolainen, T.: Discovery of geometry transition in nearby AGN jets, MNRAS, submitted, Sept. 2019.

[7] Kundt, W.: What happens at Galactic Centers? essay on gravitation, Kyoto, March 1978; received Honorable Mention; introduced 'Burning Disk'.

[8] Kundt, W.: A Model for Galactic Centers, Astrophys. and Space Science 62, 335-345, 1979.

[9] Kundt, W.: The Galactic Centre, Astrophysics and Space Science 172, 109-134, 1990. 
[10] Kundt, W.: Our Galactic Center, in: 'Jets from Stars and Galactic Nuclei', Lecture Notes in Physics 471, ed. W.Kundt, 12-18, Springer 1996.

[11] Kundt, W., Astrophysics of Neutron Stars, Fundamentals of Cosmic Physics Vol. 20, 1-119, 1998.

[12] Kundt, W.: Radio Galaxies powered by Burning Disks, in: 'Life Cycles of Radio Galaxies', New Astronomy Reviews 46, 2-7, pp. 257-261, 2002.

[13] Kundt, W., Astrophysics, A New Approach, Springer, 223 pp., 2005.

[14] Kundt, W., Our Galactic Center - the nearest Burning Disk, ACTA POLYTECHNICA 53, Supplement 2013, 506-512, 2013.

[15] Kundt, W.: Sgr A*, the best-sampled of all AGN? PoS(MULTIF 17)050, 1-12 (2017a).

[16] Kundt, W., The Astrophysical Jets (again): PoS(MULTIF 17)075, (2017b).

[17] Kundt, W., Gravitational Astrophysics: PoS(FRAPWS 2018)016, 2018.

[18] Kundt, W., Ordered Motions in the Universe, viXra:2006.0270, 2020.

[19] Kundt, W., Krause, M.: The nature of the IGM, Astron. Astrophys. 142, 150-156, 1985.

[20] Kundt, W., Marggraf, O.: Physikalische Mythen auf dem Prüfstand, Springer 2014.

[21] Kundt, W.: A brief Observational History of the Black-Hole Spacetimes, Advances in Mathematical Physics, vol. 2015, Article ID 617128, doi: 10.1155/2015/617628, 2015.

[22] Ponti, G., Hofmann, F., Churazov, E., Morris, M.R., Haberl, F., Nandra, K., Terrier, R., Clavel, M., \& Goldwurm, A.: An X-ray chimney extending hundreds of parsecs above and below the Galactic Centre, LETTER to NATURE VOL 567, 347-350, 2019, viewed by Masha Chernyakova on pp.318-320.

[23] Schaefer, Gerhard: der Quelle auf der Spur, Physik Journal 16, Nr.2, 35-40, 2017.

\section{DISCUSSION}

JIM BEALL: Narlikar suggested that white holes were responsible for AGN phenomena. Why do you believe the $\mathrm{BD}$ model is preferable?

WOLFGANG KUNDT: White holes are at least as exotic as black holes are (viewed by Einstein's GRT), whilst BDs are standard building blocks of disk theory. 